

Functional Food Science

\title{
Analysis of contemporary epidemiological study research design formats on addressing functional food efficacy
}

\author{
Pooja Agarwal', Emma Rutter ${ }^{2}$ and Danik M. Martirosyan ${ }^{3}$
}

${ }^{1}$ George Mason University, Fairfax, VA, USA; ${ }^{2}$ Santa Clara University, Santa Clara, CA, USA; ${ }^{3}$ Functional Food Center/Functional Food Institute, Dallas, TX 75252, USA

Corresponding author: Danik M. Martirosyan, PhD, Functional Food Center/Functional Food Institute, Dallas, TX 75252, USA

Submission Date: September 21 ${ }^{\text {st }}, 2021$; Acceptance Date: December 22 ${ }^{\text {nd }}, 2021$; Publication Date: December $30^{\text {th }}, 2021$

Please cite this article as: Agarwal P., Rutter E., Martirosyan D.M. Analysis of contemporary epidemiological study research design formats on addressing functional food efficacy. Functional Food Science 2021; 1(12): 97-116. DOI: https://www.doi.org/10.31989/ffs.v1i12.882

\section{ABSTRACT}

The entirety of this particular review article demonstrates an in-depth analysis of the overall functionality of nutritional functional foods, imperative bioactive compounds, and essential biomarkers within the metabolic pathway and in chronic disease progression. Specifically, this review article assesses the efficacy of a plethora of functional foods and their associated bioactive compounds via the interpretation of epidemiological research studies that were conducted in the following design formats: cohort design, case-control design, cross-sectional design, and randomized controlled trial design. Although there has been a source of ambiguity demonstrated by food scientists and government organizations surrounding what would be the standard definition of functional food, this review article attempts to advocate for a contemporary definition that acknowledges the primary attributes of present-day functional food. Having said this, the main intention of this review article is to help address the necessity of analyzing the overarching efficacy of specific functional foods via the analysis of different retrospective epidemiological studies. Moreover, this review article will acknowledge which specific epidemiological study research design format is most applicable in the evaluation of functional food products within upcoming epidemiological studies. Ultimately, food scientists and nutritionists alike will get the opportunity to develop an all-encompassing contemporary definition for functional food upon the recognition and incorporation of different epidemiological study research design formats in a timely and cost-effective manner.

KEYWORDS: Functional food, bioactive compounds, biomarkers, epidemiology, cohort design, case-control design, cross-sectional design, and randomized controlled trial design. 


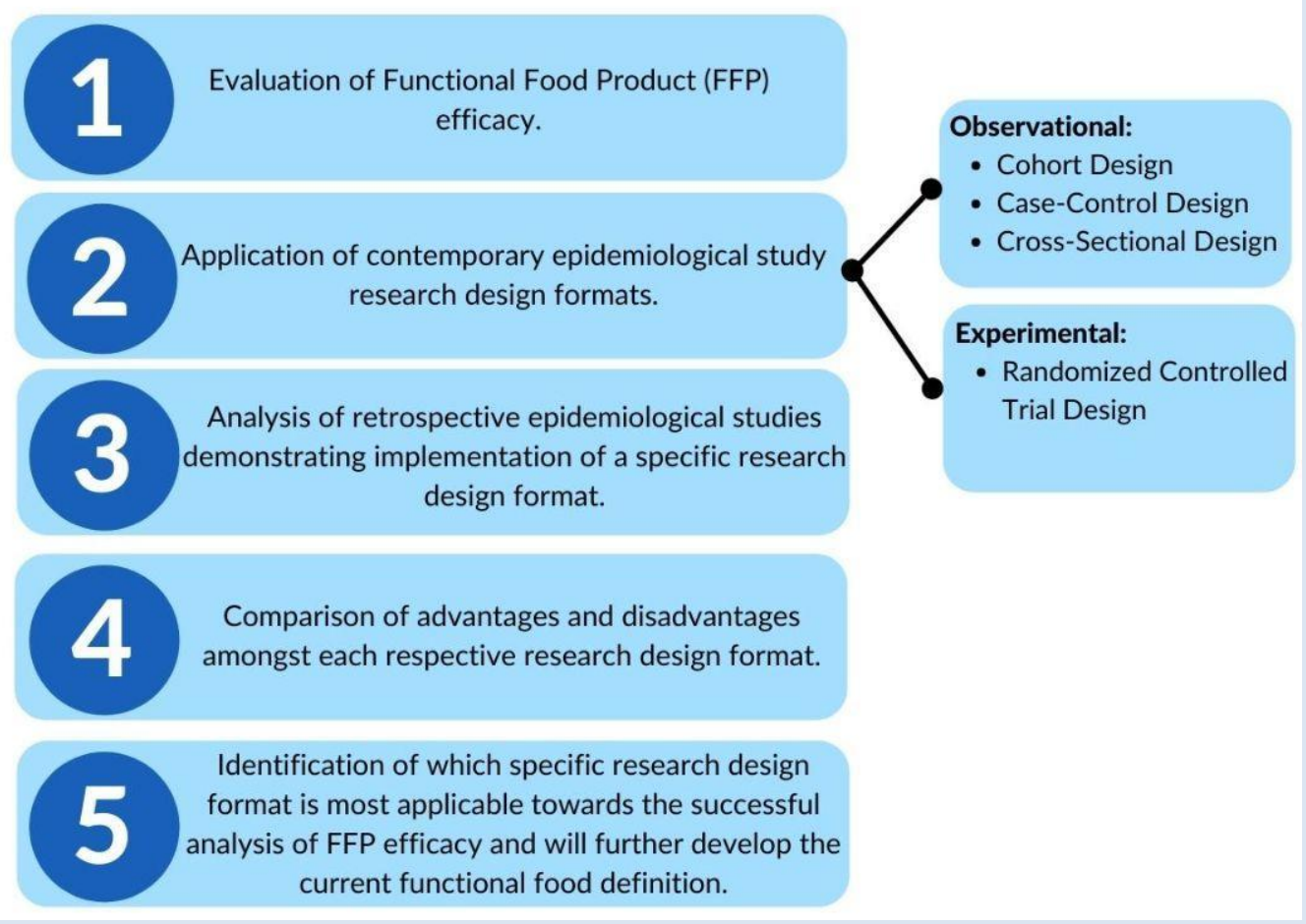

CFFC 2021. This is an Open Access article distributed under the terms of the Creative Commons Attribution 4.0 License (http://creativecommons.org/licenses/by/4.0)

\section{INTRODUCTION}

In 2009, the Functional Food Center (FFC) introduced the following concept of functional food at the 6th International Conference on Functional Food, "Natural or processed foods that contains known or unknown biologically-active compounds; which in defined amounts provide a clinically proven and documented health benefit for the prevention, management, or treatment of chronic disease" [1-2]. Research professionals emphasized that functional food is a single consumable product which can be classified as either organic or refined and is composed of a noteworthy quantitative concentration of unique bioactive compounds. Specifically, researchers acknowledged that the introduction of specific bioactive compounds at nontoxic concentrations may contribute towards controlling the chronic disease advancement [3-4].
Shortly after, the FFC organized "The Definition of Functional Foods and Bioactive Compounds" which was held at the 17th International Conference on Functional Food in 2014. One of the primary objectives of this scheduled conference was to further the definition of functional food which had been developed in 2009 by addressing competent repeatable clinical trial procedures. A mutual agreement was expressed amongby representatives from the FFC and delegates from the U.S. Department of Agriculture (USDA) and Agriculture Research Service (ARS) with regards to the following more explicit definition of functional food: "Natural or processed foods that contain known or unknown biologically-active compounds; which, in defined, effective non-toxic amounts, provide a clinically proven and documented health benefit for the prevention, management, or treatment of chronic disease." [1]. 
Advancement of Functional Food Definition: Upon comparison to the initial definition in 2009, there are a series of noteworthy revisions that were implemented within the further developed definition from 2014. Specifically, the 2014 version of the functional food definition demonstrates a keen emphasis on the necessity for incorporating a clinical research design that could be duplicated by additional food scientists and nutritionists. In other words, the 2014 definition of functional food advocates for the replication of clinical trial procedures which would be bolstered by introducing minute concentrations of a particular innocuous food. Therefore, researchers can assess the overall efficacy surrounding a research study which would consist of direct manipulation of a harmless dosage of functional food being provided to a cohort of voluntary participants. Subsequently, the 2014 variant of the functional food definition portrays the necessity of including updated data records which can potentially demonstrate the performance of a specific functional food against the overarching pathogenesis of a particular chronic disease. Analysis of retrospective case studies surrounding functional food performance can highlight the potential primary side effects and symptoms that were demonstrated by the cohort who received the treatment. Upon careful analysis of retrospective data values, researchers can interpret a potential source of correlation between the introduced dosage of functional food and chronic disease progression.

During 2018, the subsequently developed version of the functional food definition was further revised by Dr. Danik Martirosyan, President of Functional Food Center, to the following statement: "Natural or processed foods that contain biologically active compounds; which, in defined, effective non-toxic amounts, provide a clinically proven and documented health benefit utilizing specific biomarkers for the prevention, management, or treatment of chronic/viral disease or its symptoms" [5]. Upon comparison to the 2014 version, it can be said that the 2018 definition demonstrates the inclusion of implementing biomarkers in order to potentially avert, control, or remedy the continued progression of a particular chronic disease. Furthermore, the 2018 definition exhibits the acknowledgment of potentially introducing biomarkers to analyze the respective primary and secondary symptoms as presented during the gradual pathogenesis of either chronic or viral diseases.

Most recently in 2020, Dr. Danik Martirosyan further enhanced the 2018 definition by emphasizing the specialized functions of biomarkers within the progression of chronic or viral diseases in the following statement: "Natural or processed foods that contain biologically-active compounds, which, in defined, effective, non-toxic amounts, provide a clinically proven and documented health benefit utilizing specific biomarkers, to promote optimal health and reduce the risk of chronic/viral diseases and manage their symptoms" [6]. The current proposed definition of functional food fixates on the notion that the incorporation of biomarkers can potentially contribute towards improved health sustainability, diminished prospect of chronic or viral disease, and regulate the frequency at which the most prevalent symptoms are presented. Ultimately, the 2020 definition is what constitutes a functional food based on the respective functionality within the essential metabolic pathways of an individual alongside chronic or viral disease pathogenesis. However, the FFC anticipates there will be succeeding definitions developed following the 2020 definition which will be even more precise and unequivocal. Table 1 summarizes the changes of the functional food definition from 2014 to 2020. 
Table 1. Comparison of initial functional food definition to succeeding definitions as proposed by Dr. Danik Martirosyan, President of FFC, with co-authors [1-2,5-6].

\begin{tabular}{|c|c|c|c|}
\hline 2009 Definition [1] & 2014 Definition [2] & 2018 Definition [5] & 2020 Definition [6] \\
\hline $\begin{array}{l}\text { Natural or processed } \\
\text { foods } \\
\text { Known or unknown } \\
\text { bioactive compounds } \\
\text { Clinically proven } \\
\text { Documented health } \\
\text { benefit }\end{array}$ & $\begin{array}{l}\text { Natural or processed } \\
\text { foods } \\
\text { Known bioactive } \\
\text { compounds } \\
\text { Clinically proven } \\
\text { Documented health } \\
\text { benefit } \\
\text { Effective } \\
\text { Don-toxic amounts }\end{array}$ & $\begin{array}{l}\text { Natural or processed } \\
\text { foods } \\
\text { Known bioactive } \\
\text { compounds } \\
\text { Clinically proven } \\
\text { Documented health } \\
\text { benefit } \\
\text { Effective } \\
\text { Non-toxic amounts } \\
\text { Usage of biomarkers } \\
\text { Acknowledgements of } \\
\text { chronic or viral diseases }\end{array}$ & $\begin{array}{l}\text { Natural or processed } \\
\text { foods } \\
\text { Known bioactive } \\
\text { compounds } \\
\text { Clinically proven } \\
\text { Documented health } \\
\text { benefit } \\
\text { Effective } \\
\text { Non-toxic amounts } \\
\text { Usage of biomarkers } \\
\text { Advocate for improved } \\
\text { health status } \\
\text { Minimize imperil from } \\
\text { chronic or viral disease } \\
\text { progression } \\
\text { Regulate symptoms }\end{array}$ \\
\hline
\end{tabular}

Nevertheless, it remains imperative to address the historical discrepancy surrounding the establishment of an exclusive definition for functional food. In 1984, Japanese researchers from the Ministry of Health, Labor and Welfare introduced the necessity of classifying functional foods as Food for Specified Health Uses (FOSHU). The primary intention of such researchers was to implement the ideology of functional food in order to address certain chronic health conditions, mitigate micronutrient deficiency, and emphasize the importance of conducting nutritional science research in a costeffective manner. Japanese researchers defined the terminology of functional food as "Food products fortified with special constituents that possess advantageous physiological effects" [7-8]. Researchers highlighted that the foundation surrounding the primitive definition of functional food was composed based on the assurance of clinical efficacy, establishment of security, and in-depth analysis of practical bioactive constituents.
Conversely, researchers residing within the European Union further elaborated on the initially proposed definition of functional food by advocating for the analysis of the potential relationship between functional food efficacy and mitigation of the associated risk factors as a result of continued pathogenesis of a particular chronic disease, "Food products can only be considered functional if together with the basic nutritional impact it has beneficial effects on one or more functions of the human organism thus either improving the general and physical conditions or/and decreasing the risk of the evolution of diseases" [9]. Although there is no standard definition of functional food within the European Union, food scientists and associated political authorities agree over the establishment of disparate divisions within the food groups. Consequently, researchers developed the following distinguishable food categories: "conventional foods, modified foods, foods for special dietary use and medical foods" [10]. The further revised definition of functional food proposed by researchers within the European Union demonstrates 
the importance of analyzing the potential implications of functional food consumption on physiological development and regulation of essential metabolic pathways among human beings.

Whereas clinical health researchers working at the Food and Drug Administration (FDA) remain skeptical with regards to recognizing functional food as a distinct yet equally imperative food category. Such researchers prove the necessity of conducting further research with regards to assessing the respective inducing and/or inhibiting functions of bioactive compounds within central metabolic pathways of human beings who present primary and secondary symptoms of a particular chronic disease. Researchers emphasize that the overall efficacy of certain bioactive compounds may contribute towards potentially diminishing the risk of chronic disease progression which can be evaluated via the close monitoring of activated biomarkers. The FDA has not established a standard definition for functional food that has been approved by legislative and executive officials.

However, there are a series of government and private organizations within the United States that have established alternative contemporary definitions of functional food that have been collectively ratified. For instance, The Institute of Medicine of the U.S. National Academy of Sciences (NAS/IOM) further developed the definition of functional food by indicating the noteworthy health related benefits that could be associated with the regular consumption of functional foods, "Foods that encompass potentially healthful products, including any modified foods or food ingredients that may provide a health benefit beyond the nutrients it contains" [11]. Additionally, The Institute of Food Technologists (IFT) established a rather analogous definition to the definition developed by NAS/IOM which is the following: "Foods and food components that provide a health benefit beyond basic nutrition. These substances provide essential nutrients often beyond quantities necessary for normal maintenance, growth, and development, and/or other biologically active components that impact health benefits" [12].

In retrospect, the plethora of presented definitions of functional food primarily address the potential influence of specific bioactive compounds towards reducing the risk of chronic disease progression alongside the promotion of physiological development due to an enhanced establishment of nutrient intake as noted in Table 2.

Table 2. Comparison of Functional Food definitions from a global perspective [6-9,11-12].

\begin{tabular}{|c|c|}
\hline $\begin{array}{l}\text { Functional Food Center } \\
\qquad(2020)[6]\end{array}$ & $\begin{array}{l}\text { Natural or processed foods that contain biologically-active compounds which in defined, effective, } \\
\text { non-toxic amounts, provide a clinically proven and documented health benefit utilizing specific } \\
\text { biomarkers, to promote optimal health and reduce the risk of chronic/viral diseases and manage their } \\
\text { symptoms. }\end{array}$ \\
\hline $\begin{array}{l}\text { The Institute of Medicine } \\
\text { of the U.S. National } \\
\text { Academy of Sciences [11] }\end{array}$ & $\begin{array}{l}\text { Foods that encompass potentially healthful products, including any modified foods or food ingredients } \\
\text { that may provide a health benefit beyond the nutrients it contains. }\end{array}$ \\
\hline $\begin{array}{l}\text { The Institute of Food } \\
\text { Technologists [12] }\end{array}$ & $\begin{array}{l}\text { Foods and food components that provide a health benefit beyond basic nutrition. These substances } \\
\text { provide essential nutrients often beyond quantities necessary for normal maintenance, growth, and } \\
\text { development, and/or other biologically active components that impact health benefits. }\end{array}$ \\
\hline $\begin{array}{l}\text { Japan Ministry of Health, } \\
\text { Labor and Welfare [7-8] }\end{array}$ & Food products fortified with special constituents that possess advantageous physiological effects. \\
\hline European Union [9] & $\begin{array}{l}\text { Food products can only be considered functional if together with the basic nutritional impact it has } \\
\text { beneficial effects on one or more functions of the human organism thus either improving the general } \\
\text { and physical conditions or/and decreasing the risk of the evolution of diseases. }\end{array}$ \\
\hline
\end{tabular}


Nonetheless, researchers reiterate the necessity for future funded research opportunities through which the potential influence of specific bioactive compounds and related biomarkers on the pathogenesis of a particular chronic disease can be further analyzed given the condition of a long-term study. In addition, researchers indicate that additional interpretation of prospective data reports from innovative research projects may possibly contribute towards the establishment of a standard definition of functional food which can be agreed upon by health professionals, nutritionists, and policy makers globally.

Based on this notion, researchers have demonstrated a gradual transition towards the implementation of four paramount epidemiological study types, outlined in Figure 1 , in order to better understand the potential influence of specific functional foods and bioactive compounds on decreasing the risk of chronic disease advancement. This review article will analyze the efficacy of different functional foods and bioactive compounds within a spectrum of prevalent chronic diseases via the evaluation of retrospective epidemiological case studies in cohort design, casecontrol design, cross-sectional design, and randomized controlled trial design, respectively. Furthermore, this review article will ultimately determine which specific epidemiological case study types are best applicable towards analyzing the influence of various functional food products on the further prevention and/or management of chronic disease progression.

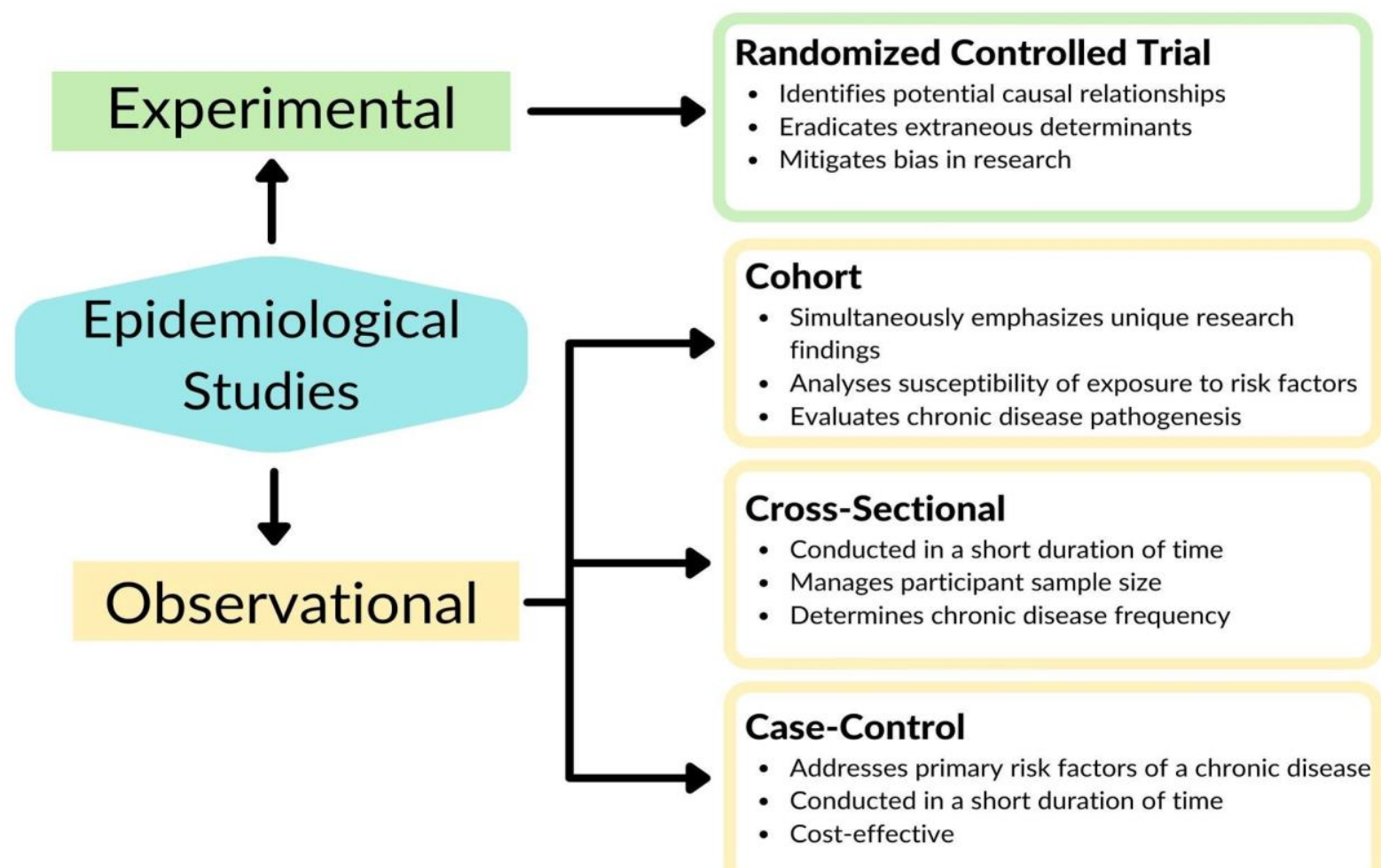

Figure 1. Description of contemporary experimental and non-experimental epidemiological research design formats [13].

Introduction to Epidemiological Studies: Epidemiological studies are categorized into two main categories, observational studies where investigators observe a particular treatment, disease or risk factor and experimental studies which allow for the assessment of a specific intervention to examine its effects. Observational studies are further divided into two subtypes: descriptive and analytic. The analytic subtype encompasses cohort studies and case-control studies. Conversely, experimental research design formats involve clinical 
studies that can be conducted with or without the application of randomization amongst disparate treatment groups. Understanding the specific characteristics of each study design, its respective advantage as well as limitation can potentially guide researchers in the correct investigative protocol for assessment of functional food and the potential to aid in the prevention, management and treatment of chronic disease.

Although there is an established distinction among the aforementioned observational and experimental research design formats, it remains imperative to acknowledge the noteworthy variation in sample sizes among an array of retrospective epidemiological studies. The composition of a satisfactory sample size is dependent on the following fundamental criteria: standard deviation, margin of error, confidence level, and power of a specific statistical test [14]. Researchers emphasize it is essential to include a sample size which will ensure a certain level of precision.

A relatively small sample size may curtail the overall precision of the collected data which can possibly influence the final acceptance or rejection of the central hypothesis statement [14]. Whereas a larger sample size provides a greater level of precision among the collected data. Larger sample sizes can be readily influenced by the access to laboratory equipment and research exclusion criteria [14]. Thus, the incorporation of a suitable sample size within epidemiological studies is essential in order to distinguish between the treatment group and the control group in an efficient and timely manner.

\section{Epidemiological Study Type I: Cohort Design}

Retrospective epidemiological studies on the assessment of functional food efficacy have exhibited an increased utilization of the cohort design to recognize the unique pathogenesis, associated symptoms, and prevalence rate for a specific chronic disease. A cohort design is recognized as an empirical study that evaluates a possible causal relationship among the manipulated treatment group and the resultant research findings over a set period of time. Based on this notion, researchers evaluate possible risk factors that may contribute to the potential escalation in risk of chronic disease progression in a chronological manner. Furthermore, researchers also determine the proportional frequency of morbidity and mortality among a population of individuals who have been exposed to explicit risk factors which could inflate the risk of chronic disease progression.

Although there are noteworthy benefits surrounding the incorporation of a cohort design within epidemiological studies, as noted in Figure 2, researchers indicate there are equally imperative disadvantages that should be taken into consideration when establishing the initial research design for epidemiological studies. For instance, cohort designs often necessitate the inclusion of a significantly broad sample size in order to successfully distinguish between the treatment group and the control group. Moreover, cohort designs are recognized as being influenced by potential research bias alongside not being conducted in a timely and costeffective manner. Nevertheless, cohort design implementation remains one of the most prevalent research design formats within retrospective epidemiological studies. Such studies recognize the possible influence of an individual's dietary and lifestyle habits alongside socioeconomic factors that further contribute towards the risk of chronic disease progression. 


\section{Cohort Design}


Figure 2. Description of the noteworthy advantages surrounding the implementation of cohort design within epidemiological studies.

In an epidemiological study conducted in March 2020, researchers from the World Cancer Research Fund (WCRF) indicated that increased consumption of fish may contribute towards a significant reduction in the susceptibility of colorectal cancer [15]. A source of ambiguity was exhibited towards the potential influence of regular consumption of specific cuts of fish, such as white or lean meat, on the risk of colorectal cancer progression. Despite this, researchers remained engrossed in the concrete analysis of the associated impact of $n-3$ polyunsaturated fatty acids ( $n-3$ LC-PUFA) which is a prevailing bioactive compound present in fish. Within this epidemiological study, researchers suggested that there is an inverse relationship present between the absorbed concentration of $n-3$ LC-PUFA and the evolution of colorectal cancer.

In a colorectal cancer study conducted in March 2020 researchers established an eight yearlong cohort research design which initially contained approximately 400,000 voluntary participants. The participants were selected based on exclusion criteria and mandatory patient screening procedures [15]. The cohort size was further reduced to 922 voluntary participants upon additional limiting factors which included body mass index (BMI), height, and dietary practices [15]. Researchers compiled blood plasma specimens from one half of the final cohort size who were diagnosed with colorectal cancer and were recognized as the treatment group. Researchers aggregated blood plasma specimens from as the control group were identified as free of colorectal cancer. Researchers provided the established treatment group with 100 grams of fish on a daily basis alongside 100 milligrams of $n-3$ LC-PUFA, while the control group did not receive any particular placebo [15]. Upon the utilization of a logistic regression model, researchers determined that there was an indirect relationship present between the regular consumption of fish and colorectal cancer risk. Researchers noted that the consumption of either lean or white cuts of fish on a weekly basis resulted in an estimated $7 \%$ decrease in the risk progression of colorectal cancer [15].

Comparatively, in a subsequent epidemiological study researchers analyzed soybean for potential influence in decreasing the risk of coronary heart disease. Researchers reported the essential amino acids in soybean elicit an overall decline in the concentrations of deleterious saturated fat and cholesterol [16]. The central purpose of this research study was to determine if there was a relationship between regular consumption of soybean and detrimental chronic diseases like obesity and cardiovascular disease. Specifically, researchers focused on the analysis of bioactive peptides, such as pepsin, which are an integral constituent found within 
the biochemical composition of soybean. The bioactive peptide named LPYPR was reported to significantly minimize the concentration of cholesterol and regulate a stable triglyceride concentration. Furthermore, researchers noted that LPYPR was in fact an antagonist to the overall functionality of the enzyme 3-hydroxy-3methylglutaryl CoA reductase (HMGR) [16]. Thus, LPYPR intervened in the activation of the HMGR enzyme which evidently inhibited cholesterol synthesis altogether.

Based on this notion, researchers established an epidemiological study with a cohort of laboratory rats. During this particular epidemiological study, researchers injected approximately 50 milligrams per kilogram of LPYPR within the laboratory rats over the time period of two consecutive days. Researchers observed an estimated $25 \%$ decrease in low-density lipoprotein (LDL) cholesterol concentration solely during this rather brief time period [16]. A steady decline in blood glucose was observed alongside an increase in insulin secretion. In a complementary epidemiological study with a cohort size of 34 female voluntary participants (age ranging from upper twenties to low forties) diagnosed with gestational diabetes, participants were asked to regularly consume soybean for 6 weeks [16]. Researchers observed a significant increase in insulin secretion and enhanced glucose concentration which notably counteracted gestational diabetes risk of progression.

Additionally, in a subsequent epidemiological study conducted in March 2018 researchers studied whether a greater consumption of pecans can directly decrease the risk of cardiovascular disease progression and the overall risk of mortality due to the presence of type II diabetes [17]. The study was designed to analyze regular pecan consumption alongside an isocaloric dietary plan and to interpret the resultant risk of cardiovascular disease and type II diabetes advancement. Researchers hypothesized pecans may decrease the risk of cardiovascular disease progression due to specific bioactive compounds like monounsaturated fatty acids [17]. The researchers also hypothesized regular consumption of pecans can successfully facilitate the overall function of lipoproteins and certain lipid concentrations found within the blood plasma given the reduced concentration of saturated fat and cholesterol, respectively [17].

Researchers established a cohort in which a total of 26 voluntary participants were selected on the basis of the following criteria: BMI, cardiovascular disease diagnosis, consumption of hormonal therapy medication, and incorporation of medicine on a daily basis to help regulate lipid activity [17]. The treatment group consisted of individuals who were diagnosed with obesity and the comorbidities of type II diabetes and cardiovascular disease. Researchers provided the treatment group with the daily recommended concentration of pecans as approved by the USDA alongside following an isocaloric dietary restriction. $t \backslash$ The control group did not receive a particular concentration of pecans and were asked to follow their daily dietary habits in order to assess for a potential influence of the placebo effect. Researchers observed a higher source of inhibition towards insulin secretion alongside the significant improvement in beta cell activity [17]. Upon the conclusion of this particular study, researchers observed a successful regulation of insulin secretion and improved beta cell functionality among the treatment group.

Researchers also noted that the regular consumption of pecans caused a decrease in low-density lipoprotein cholesterol concentration and blood glucose levels [17]. Researchers indicated that the concentration of fiber in pecans may aid in the decrease of inflammation, regulate homeostasis between glucose and insulin, and decrease physiological stress. Thus, this particular research study affirms the efficacy of pecans in successfully managing the rapid pathogenesis of cardiovascular disease. However, further research is needed. 


\section{Epidemiological Study Type II: Case-Control Design:}

The second most prevalent research design format used among retrospective epidemiological studies is a casecontrol design. This particular research design format establishes a rigid comparison among a group of individuals who have been clinically diagnosed with a particular chronic disease that pertains to the central research question of an epidemiological study to a subsequent group of individuals who have been clinically diagnosed with alternative chronic diseases. The underlying purpose behind establishing this noteworthy comparison is for researchers to get the opportunity to distinguish between the unique risk factors that potentially contribute towards the advancement of one particular chronic disease when compared to the distinct risk factors contributing towards the increase in risk of subsequent chronic diseases among a particular population of individuals.

Through the implementation of a case-control research design format, researchers are given the opportunity to identify unique research findings upon the completion of an epidemiological study. The incorporation of a case-control research design is inexpensive and can be conducted in a short amount of time which grants researchers with a greater amount of time and monetary budget that could be used towards the application of different data analysis programming languages and packages in order to assess for a potential source of association among established variables. It remains imperative to take into consideration that the implementation of a case-control research design format strictly focuses on the analysis of one particular research outcome. Case-control research design format is incapable of determining the relative incidence rate and overarching pathogenesis for a particular chronic disease. Researchers emphasize that a case-control research design format can be influenced by possible sources of research bias (Figure 3).

Although case-control design is recognized as being subordinate to cohort design in terms of providing compelling quantitative and qualitative results, researchers emphasize that a case-control design remains beneficial among epidemiological studies due to analyzing uncommon chronic diseases that are gradually increasing in prevalence on a global scale. Likewise, a case-control design is solely retrospective given that researchers initially identify a particular chronic disease among a set sample size of voluntary participants and work in reverse to identify the potential exposure determinants. By doing so, researchers not only verify specific risk factors that contribute toward the possible increase in risk of chronic disease progression but can also determine the projected incidence trends for succeeding generations.

\section{Case-Control Design}



Addresses unique research findings upon completion of epidemiological study

Figure 3. Description of the noteworthy advantages surrounding the implementation of a case-control design within epidemiological studies. 
In an epidemiological study conducted in May 2017, researchers analyzed the potential influence of dried plum (prunes), and whether consumption would mitigate the risk of osteoporosis progression [18]. Dried plums were recognized to be composed of imperative nutritional bioactive compounds, such as vitamin K, alongside a significant concentration of chlorogenic acids and phenolic compounds [18]. The phenolic compounds found within the biochemical composition of dried plums may contribute towards the fortification of bone mineral density (BMD) and would counteract the continuation of osteoporosis among postmenopausal women.

Researchers established a case-control design among a cohort of 100 postmenopausal women in which the treatment group received 100 grams of dried plum and the control group received 100 grams of dried apple [18]. Over the time duration of a year, researchers observed significant stability among the postmenopausal women who received the treatment of the dried plum when compared to the control group. Researchers noted that an increase in the frequency of dried plum consumption directly contributed towards minimizing bone deterioration and regulating BMD among postmenopausal women. Specifically, researchers observed prevention of osteoporosis progression within the ulna located in the forearm and lumbar region of the spine [18].

Subsequently, in an additional epidemiological study conducted in September 2015, researchers analyzed the efficacy of the functional food of red wine grape pomace flour in mitigating metabolic syndrome [19]. Specifically, researchers established particular interest in interpreting the functional implications of unique polyphenol compounds, which are found within wine grapes, on metabolic syndrome progression. Researchers conducted a 16-week case-control study which was composed of a cohort size of 38 voluntary participants [19]. The experimental design of this epidemiological study consisted of a treatment of 20 grams of wine grape pomace flour on a daily basis [19]. Comparatively, the established control group within this research study did not receive any particular placebo.
Upon the conclusion of the epidemiological study, researchers noted that the regular daily consumption of wine grape pomace flour contributed towards a substantial decline in systolic and diastolic blood pressure levels. More importantly, researchers reported an $8 \%$ reduction in the presentation of symptoms among the evaluated cohort of metabolic syndrome patients after the consumption of wine grape pomace flour [19]. Thus, this demonstrates that the risk of metabolic syndrome progression significantly decreased among the treatment group upon the consistent consumption of wine grape pomace flour. There were no noteworthy changes observed within the control group. Researchers encourage the regular consumption of wine grape pomace flour as this particular functional food has the capability to manage blood pressure levels and increase the concentration of gamma-tocopherol present within the blood plasma [19].

In a subsequent epidemiological study conducted in May 2019, researchers assessed whether regular ingestion of dairy based products, such as milk, contributed towards a potential decrease in the risk of bladder cancer [20]. Researchers emphasize that the primary risk factor that contributes towards the proliferation of bladder cancer risk is cigarette smoking alongside subsequent risk factors which include parasitic diseases like schistosomiasis from drinking contaminated water [20]. Researchers advocated analyzing daily dietary habits of individuals in order to measure the progression of bladder cancer and any unidentified risk factors. Researchers implemented a meta-analysis analytical method in which 18 cohort and 8 case-control study designs were evaluated [20].

The overall cohort size consisted of 595,698 voluntary participants in which 18,751 individuals were in the case-control study design and 576,946 individuals were in the cohort study design [20]. Through this 16 year epidemiological study, researchers determined that a moderate level of dairy product consumption on a daily basis contributed to a reduced risk of bladder cancer progression. Upon the application of a meta-regression model, researchers also identified that an excessive consumption of dairy products, such as whole milk, 
contributed to an increased risk of bladder cancer. Despite the completion of this particular research study, researchers demonstrate keen interest in determining whether Vitamin D levels have a potential correlation

\section{Epidemiological Study Type III: Cross-Sectional Design:}

The third most prevalent research design format used among epidemiological studies is a cross-sectional design. Cross-sectional designs consist of evaluating established variables at a specific time period; however, there is no mode of distinguishing among the possible risk factors and research outcomes. This particular research design format permits researchers to manipulate the population size being diagnosed with a specific chronic disease alongside regulation of the establishment of dependent variables within an epidemiological study. Through the implementation of a cross-sectional design researchers are able to evaluate a multitude of possible associations among the established variables in a simultaneous and timely manner (Figure 4). A cross-sectional design permits researchers to identify the prevalence rate of a specific chronic disease among a set population of individuals over a short period of time (Figure 4).

The incorporation of a cross-sectional design has noteworthy disadvantages that should be taken into with the risk of bladder cancer progression since this specific bioactive compound is recognized to facilitate cell destruction of carcinogenic cells and mitigates oncogenesis.

consideration. For example, a cross-sectional design is not able to provide statistical evidence on the overarching pathogenesis of a particular chronic disease. Furthermore, this specific research design format is unable to determine imperative research outcomes and incidence rate of a chronic disease towards the conclusion of an epidemiological study. In addition, a cross-sectional design is unable to

identify whether there is a possible relationship among established variables. Despite this, the continued implementation of cross-sectional designs in upcoming epidemiological studies remains necessary due to this particular research design format providing a platform to analyze various categorical and numerical variables in a short amount of time and in an inexpensive manner. Nonetheless, the implementation of a cross-sectional design within upcoming epidemiological studies will permit researchers to determine the prevalence rate of a specific chronic disease through which projected statistical trends can be established.

\section{Cross-Sectional Design}

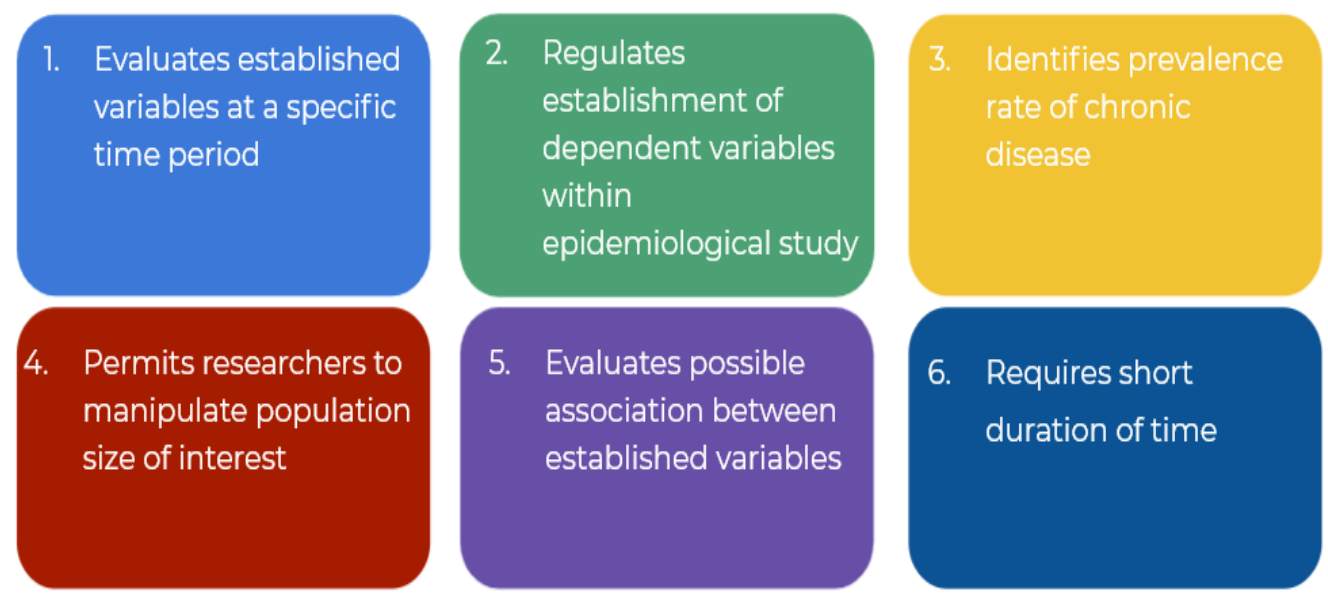

Figure 4. Description of the noteworthy advantages surrounding the implementation of a cross-sectional design within epidemiological studies. 
In an epidemiological study conducted in February 2019, researchers implemented a cross-sectional design to determine whether the daily consumption of a nutrient rich vegetative diet would readily contribute towards the mitigation of vitamin B12 deficiency [21]. Researchers established a cohort size of 103 voluntary participants from which 49 participants practiced a lactoovo vegetarian dietary habit while the remaining 54 participants regularly followed a vegan dietary habit [21]. Researchers asked the voluntary participants to regularly consume specific dairy products, such as cheese and yogurt, and closely monitored the resultant vitamin B12 and homocysteine concentrations via competitive immunoassay [21]. The researchers primarily analyzed the fluctuating concentration of the biomarker homocysteine (Hcy), in the blood plasma. Subsequently, researchers distributed a questionnaire among the collective group of voluntary participants to interpret their respective dietary habits and consumption of recommended vitamins. Researchers demonstrated a keen interest in analyzing whether a specific type of dietary habit, such as vegetarianism and veganism, could contribute towards altered vitamin B12 concentration.

Upon the conclusion of this epidemiological study, researchers noted that the concentration of homocysteine was significantly higher within the group of voluntary participants that followed a vegetarian diet in comparison to those participants who consumed a vegan diet. Specifically, researchers reported that the concentration of vitamin B12 augmented among the voluntary participants who consumed a vegetarian diet which included the consumption of yogurt. Additionally, regular consumption of yogurt among the voluntary participants who followed a vegetarian diet caused an increase in homocysteine and methylmalonic acid concentrations within the blood plasma. Researchers noted a substantial increase in the concentration of homocysteine after the consumption of egg alongside a vegetarian diet in comparison to the vegan diet.

Similarly, researchers implemented a crosssectional epidemiological study conducted in September 2019 to analyze the potential efficacy of a specific dietary mediation on metabolic syndrome progression [22]. Within this epidemiological study, researchers established a cohort size of 1065 voluntary participants who resided within Mexico [22]. The treatment group consisted of voluntary participants who were clinically diagnosed with metabolic syndrome and were asked to consume a specific dietary intervention which was low in saturated fat. The control group consisted of voluntary participants who were not diagnosed with metabolic syndrome and were asked to consume the dietary intervention that was not manipulated in order to assess the potential influence of the placebo effect. In less than half a month, researchers estimated a $24 \%$ decline in the concentration of triglycerides upon the regular consumption of the primary dietary intervention [22]. Moreover, researchers reported a decrease in lowdensity lipoprotein cholesterol levels alongside glucose concentration which readily contributed towards the management of metabolic syndrome progression.

\section{Epidemiological Study Type IV: Randomized Controlled}

Trial: The last research design format that has been recently implemented among epidemiological studies is the randomized controlled trial. This particular research design format permits researchers to successfully develop hypothesis and alternative hypotheses statements which assess a possible causal relationship among the manipulated treatment and chronic disease progression. By doing so, researchers determine whether the introduced treatment option caused a direct impact in mitigating and/or inhibiting the advancement of a particular chronic disease within a clinical setting. Additionally, this research design format encourages 
researchers to determine whether there are alternative risk factors and comorbidities that are contributing towards the continued progression of a specific chronic disease which the introduced treatment option may have not been able to successfully counteract.

Through the implementation of a randomized controlled trial design, researchers are able to collect dependable statistical evidence from the series of repeated clinical trials that are conducted within an epidemiological study. The repetition of clinical trials ensures a greater source of accuracy and overall authenticity for upcoming epidemiological studies who would want to pursue a similar mode of treatment intervention and placebo option to evaluate functional food efficacy in the prevention and/or management of a specific chronic disease. Furthermore, this particular research design format permits researchers to apply randomization within the unique treatment interventions being provided to the respective treatment groups which will readily diminish potential confounding variables that may arise within the duration of the epidemiological study. Despite the overwhelming number of advantages present for a randomized controlled trial, outlined in Figure 5, researchers should take into consideration that this specific research design format is recognized to be expensive, long term with regards to time duration, and mainly influenced by external validity.

\section{Randomized Controlled Trial Design}



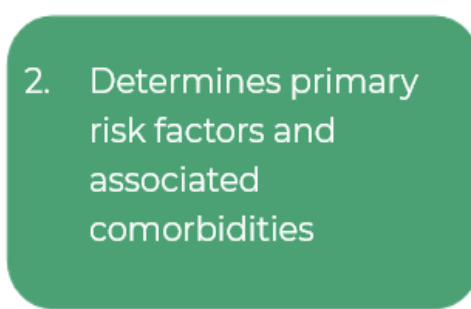

5. Incorporates a series of repeated trials

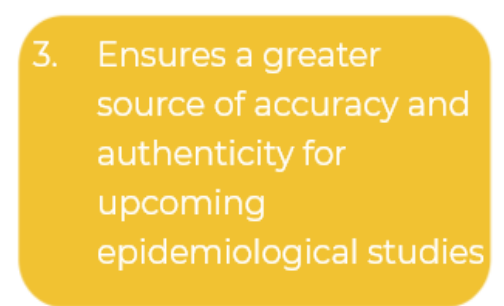

6. Implements randomization amongst treatment group

Figure 5. Description of the noteworthy advantages surrounding the implementation of a randomized controlled trial design within epidemiological studies.

In an epidemiological study conducted in September 2012, researchers implemented a randomized controlled trial design in order to analyze whether regular cinnamon ingestion at minute concentrations could successfully manage blood glucose levels among Type I and Type II diabetes mellitus patients [23]. Researchers have suggested that regular consumption of cinnamon among human beings may contribute towards the potential regulation of blood glucose levels based on a series of epidemiological studies that were conducted among a cohort of laboratory model organisms within a clinical setting. Researchers exhibited ambiguity when such clinical trials were considered to be implemented within a cohort of human beings as there were very limited research findings pertaining to the evaluation of this possible causal relationship. Therefore, researchers emphasized that regular ingestion of cinnamon may help stabilize 
blood glucose levels among human beings which will ultimately diminish the risk of ongoing chronic disease progression.

For the purposes of this epidemiological study, researchers established a cohort size of 577 voluntary participants who were between the ages of 52 to 63 years old and were diagnosed with either Type I or Type II diabetes mellitus [23]. Researchers provided the treatment group of voluntary participants with 2 grams of cinnamon (Cinnamomum spp.) per day in an encapsulated form, while the control group received no particular treatment and were tested for a potential placebo effect [23]. Towards the end of this particular 16week epidemiological study, researchers ultimately determined that there was an insignificant impact of regular cinnamon ingestion on the management of blood glucose levels among diabetes mellitus patients. Researchers emphasized the necessity of conducting further research with regards to analyzing potential risk factors and assessing possible comprehensive advantages from cinnamon ingestion. Additionally, researchers advocated for the necessity of conducting further studies surrounding the administration of cinnamon capsules alongside oral medication which may potentially help regulate blood glucose levels altogether.

Comparatively, in a supplementary epidemiological study conducted in August 2012, researchers analyzed the potential influence of cacao product consumption, such as dark chocolate, on the management of hypertension [24]. Researchers emphasized that cacao products contain flavanols. Specifically, this specific bioactive compound is recognized to stabilize blood pressure levels to a normal range and ultimately decrease the risk of cardiovascular disease progression among the adult population. This particular epidemiological study was conducted for 12 weeks with an implementation of a randomized controlled trial research design which was composed of a cohort size of
1804 voluntary participants in total [24]. The treatment group received approximately 670 milligrams of flavanols alongside 105 grams of cacao products on a daily basis, while the control group received no specific treatment [24]. Upon the conclusion of this epidemiological study, researchers noted that there was indeed a noteworthy reduction in blood pressure levels among the cohort of voluntary participants who received the cacao treatment.

\section{DISCUSSION}

This review article provides an analysis of retrospective epidemiological studies that were conducted upon the implementation of one of the following research design formats: cohort design, case-control design, crosssectional design, and randomized controlled trial design. Although each respective research study demonstrated an analysis of the overall efficacy of one particular functional food in preventing and/or managing the risk of chronic disease progression, it remains of utmost importance to recognize which specific epidemiological research design formats would be considered most applicable in the evaluation of functional food products altogether.

A cohort design presents substantial advantages and minimal disadvantages (Table 3). Identification and meticulous analysis of potential risk factors that may readily contribute towards the risk of chronic disease progression over a duration of time can be elucidated. Furthermore, the utilization of a cohort design permits researchers to gauge the prevalence of a specific chronic disease and its associated primary and secondary presented symptoms. Via the implementation of a cohort design researchers can evaluate the pathogenesis of a particular chronic disease and determine the probability for recurrence among a set population based on dietary and lifestyle habits [25-26]. Conversely, the application of a cohort design necessitates the arrangement of 
significantly large sample sizes of voluntary participants [27-28]. In addition, researchers acknowledge that cohort designs are generally neither cost effective nor brief in terms of time duration [27,29-30].

The second research design presented was a casecontrol design. The underlying function surrounding a case-control design is to make a rigid comparison between a sample size of voluntary participants who are diagnosed with a specific chronic disease and an additional sample size of voluntary participants who are not diagnosed with the unique chronic disease being analyzed [31]. In other words, a case-control design establishes a discrete comparison among individuals who present a concrete set of risk determinants of a particular chronic disease to those individuals who do not express the risk determinants related to chronic disease progression [26]. Researchers indicate that the overall foundation surrounding a case-control design is considered opposite to that of a cohort design. Specifically, a case-control design is inexpensive and transient and identifies research endpoints following the provision of a specific manipulated treatment (Table 3A).

The implementation of a case-control design demonstrates rather limited efficacy in acknowledging chronic disease pathogenesis in a chronological manner and gives rise to potential sources of research bias [30,32-34]. Researchers often tend to dismiss the application of case-control designs given the lack of risk factor identification in addition to the shortcomings to determine the projected risk of chronic disease progression among a specific population (Table 3B) [33].

The third research design format was a crosssectional design which establishes independent and dependent variables within an epidemiological study. The cross-sectional design permits researchers to analyze potential direct and/or indirect associations among a multitude of identified variables over a short duration of time $[26,35]$. Cross-sectional design provides researchers with the opportunity to determine the percent frequency of a particular chronic disease within a population at a specific time period (Table 3A). Cross-sectional designs do not establish discrepancy amongst possible risk determinants and potential causal association amongst established variables [36]. This experimental design is unable to elucidate incidence rate surrounding a particular chronic disease (Table 3B) [25,30].

The final research design format is a randomized controlled trial design. This specific research design encompasses the application of the respective steps of the scientific method in a clinical setting [37]. A randomized controlled trial design allows assessment of prophylactic effects that could contribute towards the prevention and/or management of the risk surrounding a unique chronic disease. The implementation of a randomized controlled trial design presents precise statistical research findings upon conducting a series of clinical trials and identifies causal relationships among variables that pertain to the central research question [38-40]. A randomized controlled trial design allows full randomization of voluntary participant groups which diminishes confounding variables in an epidemiological study (Table 3A) [25,41-45]. However, implementation of a randomized controlled trial design can be expensive and require an extensive input of time (Table $3 B$ ) $[30,46-$ 47].

Upon comparison of the aforementioned epidemiological research design formats, the most applicable research design format with regards to analyzing the overall efficacy of functional food products in the prevention and/or management of chronic disease progression is a randomized controlled trial design [41]. Although this research design format is recognized as expensive and longer in time duration, there is a greater likelihood that this research design format can effectively a specific functional food within particular anatomical regions and metabolic pathways of human beings $[37,48$ - 
50]. A randomized controlled trial design can readily mitigate sources of research bias and possible confounding variables. Increased application of randomized controlled trial design can aid in the development of a contemporary functional food definition that would be accepted on a global scale among food scientists, clinical researchers, and policymakers.

Table 3A. Description of the advantages of different epidemiological studies.

\begin{tabular}{|c|c|c|c|}
\hline Cohort Study & Case-Control Design & Cross-Sectional Design & $\begin{array}{c}\text { Randomized Controlled } \\
\text { Trial }\end{array}$ \\
\hline $\begin{array}{l}\checkmark \text { Identifies and analyzes the } \\
\text { potential risk factors of } \\
\text { chronic disease progression. } \\
\checkmark \text { Allows researchers to gauge } \\
\text { the prevalence of a specific } \\
\text { chronic disease and } \\
\text { symptoms. } \\
\checkmark \text { Allows for evaluation of the } \\
\text { pathogenesis of a chronic } \\
\text { disease. } \\
\checkmark \text { Determines the probability } \\
\text { for recurrence based on } \\
\text { dietary and lifestyle habits. }\end{array}$ & $\begin{array}{l}\checkmark \text { Compares individuals } \\
\text { who present a set of risk } \\
\text { determinants of a } \\
\text { particular chronic } \\
\text { disease to those } \\
\text { individuals who do not } \\
\text { express the risk } \\
\text { determinants. } \\
\checkmark \text { Identifies extraordinary } \\
\quad \text { research endpoints. } \\
\checkmark \text { Inexpensive } \\
\checkmark \text { Transient }\end{array}$ & $\begin{array}{l}\checkmark \text { Exhibits manipulation with } \\
\text { the establishment of } \\
\text { independent and dependent } \\
\text { variables. } \\
\checkmark \text { Permits researchers to } \\
\text { analyze potential } \\
\text { associations among a } \\
\text { multitude of identified } \\
\text { variables over a short } \\
\text { duration of time. } \\
\checkmark \text { Determines the percent } \\
\text { frequency of a particular } \\
\text { chronic disease within a } \\
\text { population at a specific time } \\
\text { period. }\end{array}$ & $\begin{array}{l}\checkmark \text { Shows the potential } \\
\text { prophylactic facets that } \\
\text { could contribute towards the } \\
\text { prevention and/or } \\
\text { management of the risk } \\
\text { surrounding a chronic } \\
\text { disease. } \\
\checkmark \text { Precise statistical research } \\
\text { findings. } \\
\checkmark \text { Identifies causal } \\
\text { relationships among } \\
\text { variables. } \\
\checkmark \text { Decreases potential } \\
\text { confounding variables by } \\
\text { using successful } \\
\text { randomization. }\end{array}$ \\
\hline
\end{tabular}

Table 3B. Description of the disadvantages of different epidemiological studies.

\begin{tabular}{|c|c|c|c|}
\hline Cohort Study & Case-Control Design & Cross-Sectional Design & $\begin{array}{c}\text { Randomized Controlled } \\
\text { Trial }\end{array}$ \\
\hline $\begin{array}{ll}\checkmark & \text { A large sample size of } \\
\text { voluntary participants is } \\
\text { needed } \\
\checkmark & \text { Expensive } \\
\checkmark & \text { Requires an extensive input } \\
\text { of time. }\end{array}$ & $\begin{array}{ll}\checkmark & \text { Limited efficacy in } \\
& \text { acknowledging chronic } \\
& \text { disease pathogenesis in } \\
& \text { a chronological manner } \\
\checkmark & \text { Research bias }\end{array}$ & $\begin{array}{l}\checkmark \text { Neglects the necessity for } \\
\text { establishing a discrepancy } \\
\text { among possible risk } \\
\text { determinants and research } \\
\text { conclusions. } \\
\checkmark \text { Does not highlight a } \\
\text { potential causal association } \\
\text { among established } \\
\text { variables. } \\
\checkmark \text { Unable to address a } \\
\text { noteworthy incidence rate } \\
\text { surrounding a particular } \\
\text { chronic disease. }\end{array}$ & $\begin{array}{ll}\checkmark & \text { Expensive } \\
\checkmark & \text { Requires an extensive } \\
& \text { input of time. }\end{array}$ \\
\hline
\end{tabular}




\section{CONCLUSIONS}

After a thorough analysis of each respective research design format, the randomized controlled trial design is identified as the most applicable in the development of epidemiological studies on functional food efficacy. Although the incorporation of a randomized controlled trial design is deemed as costly and time consuming, this specific research design format is the most valuable for functional food science research given there is determination of a causal relationship among established variables. Application of a randomized controlled trial design within future epidemiological studies will permit researchers to assess nutritional foods that have yet to be assigned a specific subclassification within the broad terminology of functional food.

The definition of "functional food" continues to evolve and researchers continue to assess the role of certain functional foods within the overall pathogenesis of prevalent chronic diseases. This review article describes the methodological approaches of retrospective epidemiological case studies. Upon analyzing the respective statistical findings combined with addressing the overarching research limitations within the aforementioned retrospective epidemiological studies, this review article strives to advocate for the necessity of further developing a contemporary standard definition for functional food which can be agreed upon in unison by food scientists, nutritionists, and government officials alike.

Despite a collection of retrospective epidemiological case studies analyzing the efficacy of different functional foods, there continues to remain skepticism as to the extent of a particular functional food's capability to successfully prevent and/or manage

\section{REFERENCES}

1. Martirosyan D, Singh J: A new definition of functional food by FFC: what makes a new definition unique? Functional Foods in Health and Disease 2015, 5(6): 209-223. https://doi.org/10.31989/ffhd.v5i6.183

2. Martirosyan DM, Zolaedov V, Miroshnichenko L: Functional Foods: definition and the steps necessary in developing a the risk of chronic disease progression. In conclusion, research findings provided by randomized controlled trial design are limited. The authors encourage the creation of additional funding opportunities through which subsequent epidemiological studies assessing functional food efficacy can be conducted. Additionally, the definition of "functional food" will continue to evolve as progress in research occurs.

List of Abbreviations: ARS: Agriculture Research Service, BMI: Body Mass Index, BMD: Bone Mineral Density, DMH: Dimethylhydrazine, FDA: Food and Drug Administration, FFC: Functional Food Center, FOSHU: Food for Specified Health Uses, Hcy: Homocysteine, HMGR: 3-hydroxy-3-methylglutaryl CoA reductase, LDL: Low-density lipoprotein, dp-ucMGP: Plasma DesphophoUncarboxylated, PCNA: Proliferating Cell Nuclear Antigen, n-3 LC-PUFA: n-3 Polyunsaturated Fatty Acids, IFT: The Institute of Food Technologists, NAS/IOM: The Institute of Medicine of the U.S. National Academy of Sciences, USDA: U.S. Department of Agriculture, WCRF: World Cancer Research Fund.

Acknowledgement of Competing Interests and Funding: The authors have no financial interests or any other conflicts of interest to disclose.

Authors' Contributions: Danik Martirosyan, PhD: conceived and designed the study, analyzed and interpreted the data and actively involved in writing the manuscript; Pooja Agarwal (intern student at FFC): collected the data, wrote the manuscript. Emma Rutter (intern student at FFC); assisted in editing the manuscript, created tables and figures.

successful functional food product. Proceedings of the 6th International Conference of FFC on Functional Foods for Chronic Diseases: Diabetes and Related Diseases: December 45, 2009; Texas Woman's University, Denton, TX, USA. Edited by Martirosyan DM and Prasad C, 2009:163-170.

3. Martirosyan D, Pisarski K: Bioactive Compounds: Their Role in Functional Food and Human Health, Classifications, and 
Definition. Introduction to Functional Food Science 3rd ed. 2015.

4. Gur J, Mawuntu M, Martirosyan, D. FFC's Advancement of Functional Food Definition. Functional Foods in Health and Disease 2018, 8(7): 385-397. https://doi.org/10.31989/ffhd.v8i7.531

5. Leem C, Martirosyan D: The bioactive compounds of probiotic foods/supplements and their application in managing mental disorders. Bioactive Compounds in Health and Disease 2019, 2(10): 206-220. https://doi.org/10.31989/bchd.v2i10.431

6. Martirosyan $\mathrm{D}$, Kanya $\mathrm{H}$, Nadalet $\mathrm{C}$ : Can functional foods reduce the risk of disease? Advancement of functional food definition and steps to create functional food products. Functional Food in Health and Disease 2021. 11(5): 213-221. https://www.doi.org/10.31989/ffhd.v11i5.788

7. Hardy G: Nutraceuticals and functional foods: introduction and meaning. Nutrition 2000, 16(7-8):688-9. https://doi.org/10.1016/s0899-9007(00)00332-4.

8. Kwak NS, Jukes DJ: Functional foods. Part 1: The development of a regulatory concept. Food Control 2001, 12: 99-107. https://doi.org/10.1016/S0956-7135(00)00028-1

9. Ringel Heller I, Taniguchi Y, Lobstein T: Functional foods: public health boon or 21st century quackery? International Association of Consumer Food Organizations 2015, https://doi.org/10.1136/ewjm.172.1.8

10. Office of Health Policy on Newly Developed Food in Environmental Health Bureau, Ministry of Health and Welfare: Information Regarding Permission to Use FOSHU Label 1998

11. Thomas PR, Earl R: Opportunities in the Nutrition and Food Sciences: Research Challenges and the Next Generation of Investigators. National Academies Press; 1994: 98-142. https://doi.org/10.1093/in/124.6.763

12. Institute of Food Technologists: Functional foods: Opportunities and challenges. Institute of Food Technologists 2004, 58(12):n.p.

13. Gordis, L, Friis, R, Sellers, TA: Clinical/Epidemiological Study Design. Winthrop University Hospital n.d.

14. Hajian-Tilaki K: Sample size estimation in epidemiologic studies. Caspian journal of internal medicine 2011, 2(4): 289298.

15. Aglago, EK, Huybrechts, I, Murphy, N, Casagrande, C, Nicolas, G, Pischon, T., ... Gunter, MJ: Consumption of Fish and Longchain n-3 Polyunsaturated Fatty Acids Is Associated With Reduced Risk of Colorectal Cancer in a Large European Cohort. Clinical gastroenterology and hepatology: the official clinical practice journal of the American Gastroenterological Association 2020, 18(3): 654-666.e6. https://doi.org/10.1016/j.cgh.2019.06.031

16. Chatterjee, C, Gleddie, S, Xiao, CW: Soybean Bioactive Peptides and Their Functional Properties. Nutrients 2018, 10(9). https://doi.org/10.3390/nu10091211

17. McKay, DL, Eliasziw, M, Chen, C, Blumberg, JB: A Pecan-Rich Diet Improves Cardiometabolic Risk Factors in Overweight and Obese Adults: A Randomized Controlled Trial. Nutrients 2018, 10(3): 339. https://doi.org/10.3390/nu10030339

18. Arjmandi, BH, Johnson, SA, Pourafshar, S, Navaei, N, George, KS, Hooshmand, S, Chai, SC, Akhavan, NS: Bone-Protective Effects of Dried Plum in Postmenopausal Women: Efficacy and Possible Mechanisms. Nutrients 2017, 9(5):1-19. https://doi.org/10.3390/nu9050496

19. Urquiaga, I, D’Acuna, S, Perez, D, Dicenta, S, Echeverria, G, Rigotti, A, Leighton, F: Wine grape pomace flour improves blood pressure, fasting glucose and protein damage in humans: a randomized controlled trial. Biological Research 2015, 48(1):49. https://doi.org/10.1186/s40659-015-0040-9

20. Bermejo, LM, Lopez-Plaza, B, Santurino, C, Cavero-Redondo, I, Gomez-Candela, C: Milk and Dairy Product Consumption and Bladder Cancer Risk: A Systematic Review and Meta-Analysis of Observational Studies. Advances in nutrition 2019, 10(2): 224 238. https://doi.org/10.1093/advances/nmy119

21. Gallego-Narbon, A, Zapatera, B, Barrios, L, Vaquero, MP: Vitamin B12 and folate status in Spanish lacto-ovo vegetarians and vegans. Journal of Nutritional Science 2019, 8(7):1-8. https://doi.org/10.1017/jns.2019.2

22. Guevara-Cruz, M, Flores-Lopez, AG, Aguilar-Lopez, M, SanchezTapia, M, Medina-Vera, I, Diaz, D, Tovar, AR, Torres, N: Improvement of Lipoprotein Profile and Metabolic Endotoxemia by a Lifestyle Intervention That Modifies the Gut Microbiota in Subjects With Metabolic Syndrome. Journal of the American Heart Association 2019, 8(17):1-33. https://doi.org/10.1161/jaha.119.012401

23. Leach, M. J, Kumar, S: Cinnamon for diabetes mellitus. The Cochrane database of systematic reviews 2012, (9):1-78. https://doi.org/10.1002/14651858.CD007170.pub2

24. Ried, K, Fakler, P, Stocks, NP: Effect of cocoa on blood pressure. The Cochrane database of systematic reviews 2017, 4(4). https://doi.org/10.1002/14651858.CD008893.pub3

25. Munnangi S, Boktor SW: Epidemiology Of Study Design. StatPearls Publishing 2021. 
26. Hiebert R, Nordin M: Methodological aspects of outcomes research. Eur Spine J. 2006, 15:4-16. https://doi.org/10.1007/s00586-005-1057-5

27. Nohr EA, Liew Z: How to investigate and adjust for selection bias in cohort studies. Acta Obstet Gynecol Scand 2018, 97(4):407-416. https://doi.org/10.1111/aogs.13319.

28. Rothman KJ, Greenland S, Lash TL: Modern epidemiology, 3rd edn. Lippincott Williams \& Wilkins 2008

29. DiPietro NA: Methods in epidemiology: observational study

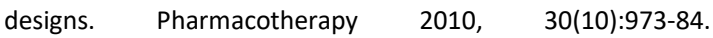
https://doi.org/10.1592/phco.30.10.973

30. Noordzij M, Dekker FW, Zoccali C, Jager KJ: Study designs in clinical research. Nephron Clin Pract. 2009, 113(3):c218-21. https://doi.org/10.1159/000235610.

31. Setia MS: Methodology Series Module 2: Case-control Studies. Indian J Dermatol 2016, 61(2):146-51. https://doi.org/10.4103/0019-5154.177773

32. Tenny S, Kerndt CC, Hoffman MR. Case Control Studies. StatPearls Publishing 2021

33. Sedgwick P: Bias in observational study designs: case-control studies. BMJ 2015, 350-560. https://doi.org/10.1136/bmj.h560

34. Groenwold RHH, van Smeden M: Efficient Sampling in Unmatched Case-Control Studies When the Total Number of Cases and Controls Is Fixed. Epidemiology 2017, 28(6):834-837. https://doi.org/10.1097/EDE.0000000000000710

35. Kesmodel US: Cross-sectional studies - what are they good for? Acta Obstet Gynecol Scand 2018, 97(4):388-393. https://doi.org/10.1111/aogs.13331

36. Chen $X$, Zhang $Z$, Yang $H$, Qiu $P$, Wang $H$, Wang $F$, Zhao Q, Fang $\mathrm{J}$, Nie J: Consumption of ultra-processed foods and health outcomes: a systematic review of epidemiological studies. Nutr J 2020, 19(1):86. https://doi.org/10.1186/s12937-020-00604-1

37. Nimavat, B. D., Zirpe, K. G., Gurav, S. K. Critical Analysis of a Randomized Controlled Trial. Indian journal of critical care medicine : peer-reviewed, official publication of Indian Society of Critical Care Medicine 2020, 24, 215-222. https://doi.org/10.5005/ip-journals-10071-23638

38. Moher D, Dulberg CS, Wells GA: Statistical power, sample size, and their reporting in randomized controlled trials. JAMA 1994, 22: $122-124$. https://doi.org/10.1001/jama.1994.03520020048013
39. Bhide A, Shah PS, Acharya G: A simplified guide to randomized controlled trials. Acta Obstet Gynecol Scand 2018, 97(4):380387. https://doi.org/10.1111/aogs.13309

40. Lim CY, In J: Randomization in clinical studies. Korean J Anesthesiol 2019, 72(3):221-232. https://doi.org/10.4097/kja.19049.

41. Lilli C, Biggeri A, Zingaretti C, Vertogen B, Frassineti V, Vespignani $R$, ... Nanni O: Is it possible to conduct clinical trials during a pandemic? The example of a trial of hydroxychloroquine. Epidemiol Prev 2021, 45(1-2):28-36. https://doi.org/10.19191/EP21.1-2.P028.036

42. Patino $\mathrm{CM}$, Ferreira JC: Internal and external validity: can you apply research study results to your patients? J Bras Pneumol 2018, 44(3):183. https://doi.org/10.1590/S1806-37562018000000164.

43. Sil A, Kumar P, Kumar R, Das NK: Selection of Control, Randomization, Blinding, and Allocation Concealment. Indian Dermatol Online J 2019, 10(5):601-605. https://doi.org/10.4103/idoj.IDOJ 14919

44. Tanniou J, van der Tweel I, Teerenstra S, Roes KC: Subgroup analyses in confirmatory clinical trials: time to be specific about their purposes. BMC Med Res Methodol 2016, 16:20. https://doi.org/10.1186/s12874-016-0122-6

45. Burke JF, Sussman JB, Kent DM, Hayward RA: Three simple rules to ensure reasonably credible subgroup analyses. BMJ 2015, 351:h5651. https://doi.org/10.1136/bmj.h5651

46. Smith GC, Pell JP: Parachute use to prevent death and major trauma related to gravitational challenge: systematic review of randomised controlled trials. BMJ 2003, 327:1459-61. https://doi.org/10.1136/bmj.327.7429.1459

47. Hlatky MA, Owens DK, Sanders GD: Cost-effectiveness as an outcome in randomized clinical trials. Clin Trials 2006, 3(6):54351. https://doi.org/10.1177/1740774506073105

48. Lachin JM, Matts JP, Wei L: Randomization in clinical trials: conclusions and recommendations. Control Clin Trials 1988, 9:365-74. https://doi.org/10.1016/0197-2456(88)90049-9

49. Schulz KF, Grimes DA: Unequal group sizes in randomised trials: guarding against guessing. Lancet 2002, 359: 966-70. https://doi.org/10.1016/S0140-6736(02)08029-7

50. Schulz KF: Randomized controlled trials. Clin Obstet Gynecol 1998, 41: 245-56. https:/doi.org/10.1097/00003081$\underline{199806000-00005}$ 